\title{
ABC Spotlight on emerging microRNA analysis methods
}

\author{
Adam T. Woolley ${ }^{1}$
}

Received: 21 May 2015 / Accepted: 26 May 2015 / Published online: 9 June 2015

(C) Springer-Verlag Berlin Heidelberg 2015

MicroRNAs (miRNAs) are short ( 20-base) non-coding RNA sequences that are biochemical modulators of gene expression, influencing development, cell proliferation, apoptosis, and other processes [1]. As of 2013, nearly 2000 human miRNA sequences had already been identified [2]. Importantly, changes in the levels of miRNAs in many diseases, combined with the presence of these miRNAs in biological fluids (blood, urine, cerebrospinal fluid, etc.), make them promising biomarkers for a variety of diseases [1]. Leuenberger et al. [2] further described the potential of miRNAs as biomarkers in the fight against doping in athletics. Accurate determination of the concentrations of specific miRNA sequences in clinical and biological samples is thus important. However, miRNAs have a number of intrinsic characteristics, including low concentrations (pM to $\mathrm{fM}$ ), short lengths, sequence similarity, and difficulty in selective amplification, all of which make the accurate quantitation of miRNAs challenging [1]. Interestingly, the most prevalent method for studying miRNA, quantitative reverse-transcription stem-loop PCR, is somewhat of a misnomer because it does not actually quantify miRNA concentration. Instead, it determines the cycle of quantitation (Cq), or the number of thermal cycles in PCR needed to confirm specific amplification. Thus, relative values such as $\mathrm{Cq}$ or related measures for miRNAs, instead of actual concentrations, are typically found in the literature. Clearly, the development of improved methods for measuring the concentrations of miRNAs in clinical and biological specimens is an important challenge for the bioanalytical chemistry

Adam T. Woolley

atw@byu.edu

1 Department of Chemistry and Biochemistry, Brigham Young University, Provo, UT 84602, USA community. This Spotlight focuses on recent advances in miRNA analysis, including sample preparation methods and detection approaches.

A key challenge in the detection of miRNAs is sample preparation because miRNA represents a very small part of the RNA content in biological samples; for example, miRNA comprises about $0.01 \%$ of the total RNA in cells [3]. A 2-fold increase in the quantity of miRNA recovered from cells was reported when a nonionic surfactant (Triton $\mathrm{X}-100$ ) was used in conjunction with the standard detergents in a commercial miRNA preparation system (TRIzol, Life Technologies, Grand Island, NY) [3]. The improved miRNA extraction was verified through capillary electrophoresis analysis of miRNA-21 extracted from $\mathrm{H} 9 \mathrm{c} 2$ and HeLa cells. In body fluid samples, such as urine or blood, miRNA is found in exosomes, lipid bilayer vesicles that are usually $<100 \mathrm{~nm}$ in diameter and are transferred from cells into the extracellular space. Exosomes are typically purified through differential ultracentrifugation, which can be both time consuming and labor intensive, such that improved exosome preparation strategies are of interest. Gale et al. [4] recently demonstrated the use of field flow fractionation methods to separate exosomes derived from mouse melanoma cultured cells. They found that exosome subpopulations could be separated without immunoaffinity or other labeling steps that are typically used but complicate the process. Field flow fractionation is also an approach that can be adapted for the preparative scale, potentially improving throughput and reducing the time needed to collect exosome fractions.

Because of challenges associated with the quantitation of miRNA, numerous strategies are being pursued to provide sequence-selective and simple quantitation of miRNA. One emerging approach involves sequence-specific hybridization of miRNA to a complementary locked nucleic acid oligonucleotide probe, followed by treatment with poly(U) 
polymerase that generates pyrophosphate, which is then detected via luciferin/luciferase luminescence [5]. This method was evaluated on synthetic miRNA sequences and had a detection limit of $50 \mathrm{fM}$, well within the relevant range for clinical samples. The ability to detect multiple miRNA sequences in a microarray format was also demonstrated. Electrochemical techniques are especially attractive for the measurement of miRNA because they can have low detection limits and provide simplicity that is desirable for point-of-care assays [6]. In one approach, miRNA-222, which is implicated in several cancers, was quantified by hybridization with a capture sequence on magnetic beads, followed by enzymatic steps that generated a molecular product that was detected at an electrode [7]. This method provided detection limits in the low pM concentration range. The same electrochemical assay was also performed in an eight-channel microfluidic format, demonstrating potential for multiplexing. In another electrochemical method for miRNA detection, capture oligonucleotides were conjugated to a conductive polymer substrate, and squarewave voltammetry was then used to probe for hybridization events [8]. Current at the conductive electrode increased upon specific hybridization, which resulted in a more organized, double-stranded structure that increased the ionic flux to the electrode. Detection limits below $1 \mathrm{pM}$ were obtained for a set of three cancer-related miRNAs. Oishi [9] recently demonstrated a non-enzymatic approach for miRNA analysis. The miRNA was hybridized with oligonucleotides that were ligated via a "click chemistry" reaction, which then facilitated a hybridization chain reaction cascade that was read out fluorescently. Limits of detection in the low pM concentration range were obtained in a matrix of diluted fetal bovine serum.

An emerging area of interest in miRNA analysis is the use of microfluidic systems, which facilitate small volume and portable assays. Indeed, a microfluidic platform was an integral part of an electrochemical miRNA detection method described above [7]. A recent miRNA analysis approach utilized the mixed-phase droplet microfluidic system shown in Fig. 1 to carry out all mixing and reaction steps in aqueous droplets isolated in an immiscible carrier fluid to determine concentrations of miRNAs in the $\mathrm{nM}$ to $\mathrm{pM}$ range [10]. Sequences of miRNAs related to hypoxia in cultured cells and DNA analogues of miRNA from genetically modified soybeans were detected in this microfluidic system. In another strategy, Chatterjee et al. [11] measured flow distances in deformable microfluidic channels coated with receptors to a target nucleic acid to determine concentrations of DNA analogues of miRNAs. Detection limits in the low pM range with single-base mismatch sequence specificity were obtained. The ability to detect these nucleic acids in a urine matrix was also demonstrated with an assay time of a few minutes in a format that was readily adaptable to point-of-care usage.

The papers described in this Spotlight highlight a number of key advances that address the limitations of the widely used but imperfect reverse-transcription stem-loop PCR method for miRNA analysis. As improved techniques for miRNA determination continue to be developed, the ability to clearly link these miRNA biomarkers to diseases will be enhanced, leading to better medical care. Especially valuable will be methods that focus on simple, quantitative, and inexpensive assays, with emphasis on capabilities such as those provided by microfluidics that can be used for pointof-care miRNA determinations. The journal Analytical and Bioanalytical Chemistry will continue to have strong interest in publishing such improvements that address existing limitations of microRNA analysis.
Fig. 1 (a) Microfluidic device used for droplet-based nucleicacid detection. (b) Representative optical image of a droplet generated in the microfluidic device. Reprinted with permission from [10]

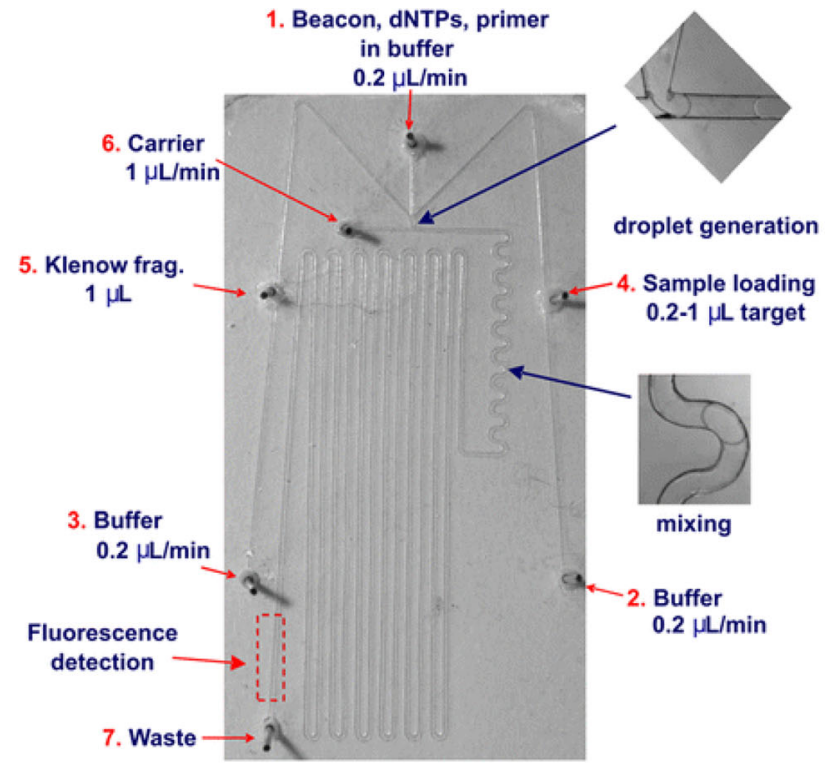

(a)

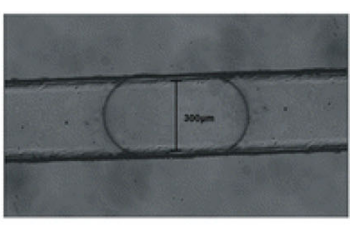

(b) 


\section{References}

1. Dong H, Lei J, Ding L, Wen Y, Ju H, Zhang X (2013) MicroRNA: function, detection, and bioanalysis. Chem Rev 113:6207-6233

2. Leuenberger N, Robinson N, Saugy M (2013) Circulating miRNAs: a new generation of anti-doping biomarkers. Anal Bioanal Chem 405:9617-9623

3. Ban E, Chae D-K, Song EJ (2013) Enhanced extraction efficiency of miRNA from cells by addition of Triton X-100. Anal Bioanal Chem 405:7535-7539

4. Petersen KE, Manangon E, Hood JL, Wickline SA, Fernandez DP, Johnson WP, Gale BK (2014) A review of exosome separation techniques and characterization of B16-F10 mouse melanoma exosomes with AF4-UV-MALS-DLS-TEM. Anal Bioanal Chem 406:7855-7866

5. Ma C, Yeung ES, Qi S, Rui H (2012) Highly sensitive detection of microRNA by chemiluminescence based on enzymatic polymerization. Anal Bioanal Chem 402:2217-2220

6. Campuzano S, Pedrero M, Pingarrón JM (2014) Electrochemical genosensors for the detection of cancer-related miRNAs. Anal Bioanal Chem 406:27-33

7. Bettazzi F, Hamid-As1 E, Esposito CL, Quintavalle C, Formisano N, Laschi S, Catuogno S, Iaboni M, Marrazza G, Mascini M, Cerchia L, De Franciscis V, Condorelli G, Palchetti I (2013) Electrochemical detection of miRNA-222 by use of a magnetic bead-based bioassay. Anal Bioanal Chem 405:1025-1034

8. Tran HV, Piro B, Reisberg S, Anquetin G, Duc HT, Pham MC (2014) An innovative strategy for direct electrochemical detection of microRNA biomarkers. Anal Bioanal Chem 406: $1241-1244$
9. Oishi M (2015) Enzyme-free and isothermal detection of microRNA based on click-chemical ligation-assisted hybridization coupled with hybridization chain reaction signal amplification. Anal Bioanal Chem. doi:10.1007/s00216-015-8629-y

10. Giuffrida MC, Zanoli LM, D'Agata R, Finotti A, Gambari R, Spoto G (2015) Isothermal circular-strand-displacement polymerization of DNA and microRNA in digital microfluidic devices. Anal Bioanal Chem 407:1533-1543

11. Chatterjee D, Mansfield DS, Woolley AT (2014) Microfluidic devices for label-free and noninstrumented quantitation of unamplified nucleic acids by flow distance measurement. Anal Methods 6 : 8173-8179

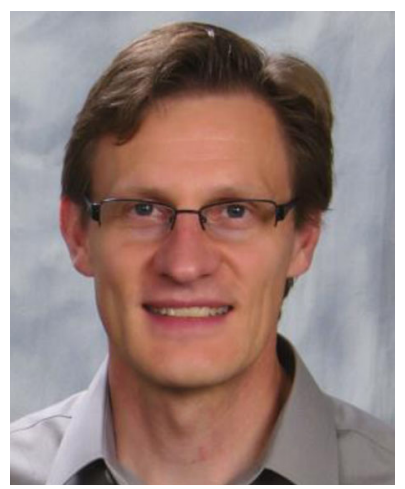

Adam T. Woolley is Professor and Associate Chair in the Department of Chemistry and Biochemistry at Brigham Young University in Provo, Utah, USA. He is the recipient of a Presidential Early Career Award for Scientists and Engineers (2006) and the ACS Division of Analytical Chemistry Award for Young Investigators in Separation Science (2007). His current research focuses on three general topics: the development of novel and sophisticated integrated microfluidic systems for preterm birth biomarker quantitation, the design of simple miniaturized biomolecular assays, and biotemplated fabrication of nanoelectronic systems. 